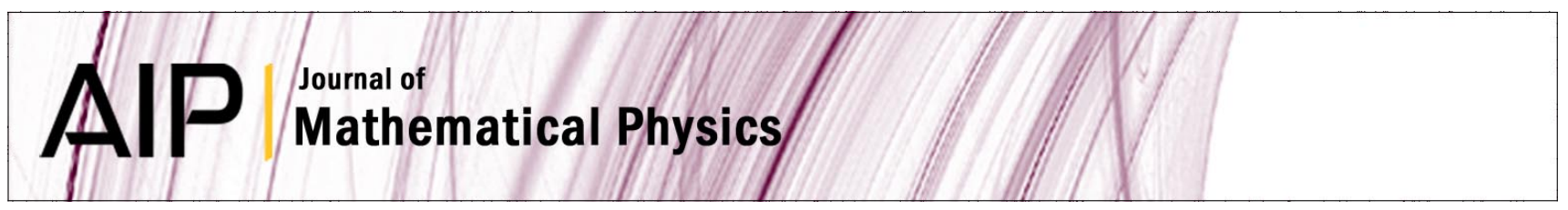

\title{
Existence of ground states for a one-dimensional relativistic Schrödinger equation
}

Juan P. Borgna and Diego F. Rial

Citation: J. Math. Phys. 53, 062301 (2012); doi: 10.1063/1.4726198

View online: http://dx.doi.org/10.1063/1.4726198

View Table of Contents: http://jmp.aip.org/resource/1/JMAPAQ/v53/i6

Published by the American Institute of Physics.

\section{Related Articles}

Communication: Phase space wavelets for solving Coulomb problems

J. Chem. Phys. 137, 101103 (2012)

The spectral shift function and Levinson's theorem for quantum star graphs

J. Math. Phys. 53, 082110 (2012)

Strong curvature effects in Neumann wave problems

J. Math. Phys. 53, 083507 (2012)

The Klein-Gordon equation with ring-shaped potentials: Asymptotic iteration method

J. Math. Phys. 53, 082107 (2012)

Two-dimensional magnetic Schrödinger equations, Darboux transformations and solutions of associated auxiliary equations

J. Math. Phys. 53, 082108 (2012)

\section{Additional information on J. Math. Phys.}

Journal Homepage: http://jmp.aip.org/

Journal Information: http://jmp.aip.org/about/about_the_journal

Top downloads: http://jmp.aip.org/features/most_downloaded

Information for Authors: http://jmp.aip.org/authors

\section{ADVERTISEMENT}

The most comprehensive support for physics in any mathematical software package World-leading tools for performing calculations in theoretical physics

Your work in Maple matches how you would write the problems and solutions by hand

- State-of-the-art environment for algebraic computations in physics

The only system with the ability to handle a wide range of physics computations as well as pencil-and-paper style input and textbook-quality display of results

- Access to Maple's full mathematical power, programming language, visualization routines, and document creation tools 


\title{
Existence of ground states for a one-dimensional relativistic Schrödinger equation
}

\author{
Juan P. Borgna ${ }^{1, a)}$ and Diego F. Rial ${ }^{2, b)}$ \\ ${ }^{1}$ Instituto de Ciencias, Universidad Nacional de General Sarmiento, Juan María Gutierrez \\ 1150, Los Polvorines, Pcia de Bs. As, 1613 Argentina \\ ${ }^{2}$ Dpto. de Matemática, IMAS-CONICET, FCEyN, Universidad de Buenos Aires, Intendente \\ Güiraldes 2160, Ciudad Universitaria, Pabellón I, Buenos Aires C1428EGA, Argentina
}

(Received 24 August 2011; accepted 18 May 2012; published online 14 June 2012)

Relativistic Schrödinger equation with a nonlinear potential interaction describes the dynamics of a particle, with rest mass $m$, travelling to a significant fraction $|v|<1$ of the light speed $c=1$. At first, we deal with the local and global existence of solutions of the flux, and in the second term, and according to the relativistic nature of the problem, we look for boosted solitons as $\psi(x, t)=e^{i \mu t} \varphi_{v}(x-v t)$, where the profile $\varphi_{v} \in H^{1 / 2}(\mathbb{R})$ is a minimizer of a suitable variational problem. Our proof uses a concentration-compactness-type argument. Stability results for the boosted solitons are established. (C)2012 American Institute of Physics. [http://dx.doi.org/10.1063/1.4726198]

\section{INTRODUCTION}

In this paper, we study the existence of solutions of the potential nonlinear relativistic Schrödinger equation, for the critical case. For the one-dimensional case, this is the cubic equation given by

$$
i \partial_{t} \psi=\left(\sqrt{-\partial_{x}^{2}+m^{2}}-m\right) \psi-|\psi|^{2} \psi,
$$

where $m \geq 0$ is the rest mass of a relativistic particle. Operator $\sqrt{-\partial_{x}^{2}+m^{2}}$ is defined via its symbol $\sqrt{\xi^{2}+m^{2}}$ under Fourier transform. The nonlinear term represents a self-interaction of the charge density. With this type of nonlinearity, Eq. (1) is a relativistic equation because the nonlinear term is Lorentz covariant as well as its linear part, this is a significant difference with the semi-relativistic equation given in Refs. 5 and 6, where the authors deal with a Hartree's type nonlinear term. In this work, they take advantage of the smoothing effect of this kind of nonlinearity with the aim to establish the existence of ground states. Also, in a recent paper, ${ }^{1}$ its authors study another semirelativistic equation, they deal with the Scrhödinger-Poisson-Slater equation, which arises in the approximation of the Hartree-Fock model for $\mathrm{N}$ particles. They have a nonlinearity with two terms, a Hartree's type as the first one and as a second term a potential nonlinearity. By effect of this last term, they only rely on a slightly smoothing effect due to the Hartree nonlinearity, and they require a new technique in order to prove the existence of the, nonboosted, ground state. In neither of the two previous works cited, the authors discuss the local and global existence of the flux of their respective equations.

At the first part of this work, we deal with the well posedness of the initial problem associated with Eq. (1). The first result is the local existence in the time, of solutions of Eq. (1) in the space $H^{s}(\mathbb{R})$, with $s>1 / 2$, for initial data $\psi_{0}(x) \in H^{s}(\mathbb{R})$. In a second step, we prove global existence

\footnotetext{
a)Electronic mail: jpborgna@ungs.edu.ar.

b) Electronic mail: drial@dm.uba.ar.
} 
in the time of these solutions, if the charge density of the initial data $\psi_{0}(x)$ is small enough. An estimation of the bound of $\left\|\psi_{0}\right\|_{2}^{2}$, under which global existence is guaranteed, is provided.

Throughout this work, we use profusely that the density of charge $\mathcal{N}(\psi)=\|\psi\|_{2}^{2}$ and the energy functional

$$
\mathcal{E}(\psi)=\frac{1}{2} \int_{\mathbb{R}} \psi^{*}\left(\sqrt{-\partial_{x}^{2}+m^{2}}-m\right) \psi d x-\frac{1}{4} \int_{\mathbb{R}}|\psi|^{4} d x
$$

are conserved along the flux of Eq. (1).

In the second part of this paper, we study solitary wave solutions of Eq. (1). By the focusing nature of the nonlinearity in (1), there exist ground states solutions. In addition, due to the relativistic nature of the problem, and taking into account the need of the fulfilment of the Lorentz's invariance of the solution, we propose, such as in Ref. 5, a ground state solution as a boosted solution, i.e.,

$$
\psi(t, x)=e^{i t \mu} \varphi_{v}(x-v t)
$$

where $|v|<1$ is the normalize velocity of the particle ( $v=1$ is the light speed) and $\mu \in \mathbb{R}$ is a phase parameter. Plugging ansatz (3) into Eq. (1), we obtain the equation that has to satisfy the profile $\varphi_{v}(x)$ of the ground state given in (3),

$$
\left(\sqrt{-\partial_{x}^{2}+m^{2}}-m\right) \varphi_{v}+i v \partial_{x} \varphi_{v}-\left|\varphi_{v}\right|^{2} \varphi_{v}=-\mu \varphi_{v}
$$

which is the Euler-Lagrange's equation for the following functional and its related constrained minimization problem

$$
\begin{aligned}
& \mathcal{E}_{v}(\varphi)=\frac{1}{2}\left\langle\varphi,\left(\sqrt{-\partial_{x}^{2}+m^{2}}-m+i v \partial_{x}\right) \varphi\right\rangle-\frac{1}{4} \int_{\mathbb{R}}|\varphi|^{4} d x \quad \text { such that } \mathcal{N}(\varphi)=N, \\
& E_{v}(N)=: \inf \left\{\mathcal{E}_{v}(\varphi) / \varphi \in H^{1 / 2}(\mathbb{R}), \mathcal{N}(\varphi)=N\right\} .
\end{aligned}
$$

From now on, we call a minimizer $\varphi_{v} \in H^{1 / 2}(\mathbb{R})$ of (6) as the profile of a boosted ground state. One of the goals of this paper is to prove the existence of solutions of the constrained problem (4), by solving minimization problem (6).

At the last part of this work, we introduce a weak orbital stability concept and we check that the flux of Eq. (1) verifies it.

\section{PRELIMINARIES}

For $s \in \mathbb{R}$, we introduce fractional Sobolev spaces

$$
H^{s}(\mathbb{R})=\left\{\psi \in \mathcal{S}^{\prime}(\mathbb{R}):\left(1+\xi^{2}\right)^{s / 2} \hat{\psi} \in L^{2}(\mathbb{R})\right\}
$$

where $\hat{\psi}$ is the Fourier transform of the function $\psi \in \mathcal{S}^{\prime}(\mathbb{R})$ (space of temperate distributions). Also we will considerer the inner product in $H^{s}(\mathbb{R})$ given by

$$
\langle\varphi, \psi\rangle_{H^{s}}=\operatorname{Re}\left(\int\left(1+\xi^{2}\right)^{s} \hat{\varphi}^{*}(\xi) \hat{\psi}(\xi) d \xi\right) .
$$

Notice that $H^{s}(\mathbb{R})$ is a real Hilbert space and, if $s>1 / 2, \hat{\psi} \in L^{1}(\mathbb{R})$, thus $\psi \in C_{0}(\mathbb{R})$.

We define the following operators, with domain in $H^{s}(\mathbb{R})$ for $s \geq 1 / 2$, and their symbols under Fourier transform:

$$
\begin{gathered}
D \psi=\sqrt{-\partial_{x}^{2}} \psi \rightleftarrows|\xi| \hat{\psi}, \\
L_{0} \psi=\left(D+i v \partial_{x}\right) \psi \rightleftarrows(|\xi|-v \xi) \hat{\psi}
\end{gathered}
$$




$$
\begin{aligned}
J \psi & =\sqrt{-\partial_{x}^{2}+m^{2}} \psi \rightleftarrows \sqrt{m^{2}+\xi^{2}} \hat{\psi}, \\
L_{m} \psi & =(J-m I) \psi+i v \partial_{x} \psi \rightleftarrows\left(\left(\sqrt{m^{2}+\xi^{2}}-m\right)-v \xi\right) \hat{\psi},
\end{aligned}
$$

and their corresponding quadratic forms

$$
\begin{aligned}
\langle D \psi, \psi\rangle & =\int_{\mathbb{R}}|\xi||\hat{\psi}|^{2} d \xi, \\
\left\langle L_{0} \psi, \psi\right\rangle & =\int_{\mathbb{R}}(|\xi|-v \xi)|\hat{\psi}|^{2} d \xi, \\
\langle J \psi, \psi\rangle & =\int_{\mathbb{R}} \sqrt{m^{2}+\xi^{2}}|\hat{\psi}|^{2} d \xi, \\
\left\langle L_{m} \psi, \psi\right\rangle & =\int_{\mathbb{R}}\left(\sqrt{m^{2}+\xi^{2}}-m-v \xi\right)|\hat{\psi}|^{2} d \xi .
\end{aligned}
$$

In the following propositions, we present useful estimations in $H^{s}(\mathbb{R})$.

Proposition 1: If $s>1 / 2$, there exists $c_{s}>0$ such that if $\psi \in H^{s}(\mathbb{R})$ it verifies

$$
\|\psi\|_{\infty} \leq c_{s}\left(1+\|\psi\|_{H^{1 / 2}} \sqrt{\log \left(2+\|\psi\|_{H^{s}}\right)}\right) .
$$

Proof: It is a special case of a general result (see Refs. 4 or 8).

Proposition 2: If $s \geq 0$, there exists $c_{s}>0$ such that if $\varphi, \psi \in H^{s}(\mathbb{R}) \cap L^{\infty}(\mathbb{R})$ it verifies

$$
\|\varphi \psi\|_{H^{s}} \leq c_{s}\left(\|\varphi\|_{H^{s}}\|\psi\|_{\infty}+\|\varphi\|_{\infty}\|\psi\|_{H^{s}}\right) .
$$

As a special case, if $s>1 / 2$ we have the following algebra structure:

$$
\|\varphi \psi\|_{H^{s}} \leq c_{s}\|\varphi\|_{H^{s}}\|\psi\|_{H^{s}} .
$$

Proof: See Ref. 8.

Proposition 3: If $\chi \in C_{0}^{\infty}(\mathbb{R})$, then $\left[L_{0}, \chi\right] \in \mathcal{B}\left(L^{2}(\mathbb{R})\right)$ and

$$
\left\|\left[L_{0}, \chi\right] \psi\right\|_{2} \leq C\left\|\partial_{x} \chi\right\|_{A}\|\psi\|_{H^{1 / 2}}^{2} .
$$

Proof: See. Ref. 8.

Now, we present a very important estimation of the norm in $L^{4}(\mathbb{R})$ by the product of the semi-norm $(9 \mathrm{a})$ and the norm $L^{2}(\mathbb{R})$.

Proposition 4: Given $\psi \in H^{1 / 2}(\mathbb{R})$, it verifies that

$$
\|\psi\|_{4}^{4} \leq \frac{8}{\pi}\langle\psi, D \psi\rangle\|\psi\|_{2}^{2} .
$$

Proof: By Hausdorf-Young's inequality (see Ref. 9) we have that

$$
\|\psi\|_{4} \leq(2 \pi)^{-1 / 4}\|\hat{\psi}\|_{4 / 3} .
$$

If $R>0$, applying Hölder's inequality we have that

$$
\begin{aligned}
& \int_{|k|<R}|\hat{\psi}(k)|^{4 / 3} d k \leq(2 R)^{1 / 3}\left(\int_{|k|<R}|\hat{\psi}(k)|^{2} d k\right)^{2 / 3} \\
& \int_{|k| \geq R}|\hat{\psi}(k)|^{4 / 3} d k \leq\left(\frac{2}{R}\right)^{1 / 3}\left(\int_{|k| \geq R}|k||\hat{\psi}(k)|^{2} d k\right)^{2 / 3} .
\end{aligned}
$$


Taking $R=\left\|D^{1 / 2} \psi\right\|_{2}^{2} /\|\psi\|_{2}^{2}$, we obtain

$$
\|\hat{\psi}\|_{4 / 3}^{4 / 3} \leq 2^{4 / 3}\left\|D^{1 / 2} \psi\right\|_{2}^{2 / 3}\|\psi\|_{2}^{2 / 3}
$$

therefore, using this estimation into inequality (12). we obtain (11).

At last, we introduce a Gronwall's type lemma.

Lemma 5: Let $a>0$ be, and an increasing and positive function $f \in C(0,+\infty)$, such that $\int_{a}^{\infty} 1 / f(\eta) d \eta=+\infty$. If $u \in C[0, T], u(t)>0$, such that it verifies the following inequality:

$$
u(t) \leq a+\int_{0}^{t} f(u(\tau)) d \tau
$$

then there exists an increasing function $G_{a} \in C^{1}[0,+\infty)$, with $G_{a}(0)=$ a such that

$$
u(t) \leq G_{a}(t) \quad \text { for all } t \in[0, T] .
$$

Proof: It is an elementary calculus.

\section{EVOLUTION PROBLEM}

\section{A. Generalization to pseudo-differential operators}

Let $M: \mathbb{R}^{n} \rightarrow \mathbb{C}$, a function that satisfies the following property:

$$
0<\liminf _{|\xi| \rightarrow \infty} \frac{|M(\xi)|}{|\xi|} \leq \limsup _{|\xi| \rightarrow \infty} \frac{|M(\xi)|}{|\xi|}<\infty
$$

then we define a pseudo-differential operator $L$ as

$$
\widehat{L \psi}=M \hat{\psi} .
$$

If $\gamma \in \mathbb{R}$, we can consider the following general pseudo-differential Cauchy problem:

$$
\begin{aligned}
i \psi_{t} & =L \psi-\gamma|\psi|^{2} \psi, \\
\left.\psi\right|_{t=0} & =\psi_{0} .
\end{aligned}
$$

From condition (13), it is easy to check that there exist numbers $0<a \leq b$ such that $a \sqrt{1+\xi^{2}}$ $\leq|M(\xi)| \leq b \sqrt{1+\xi^{2}}$, then we have that the pseudo-differential operator $L$ verifies

$$
a\|\psi\|_{H^{1 / 2}}^{2} \leq\langle\psi, L \psi\rangle \leq b\|\psi\|_{H^{1 / 2}}^{2} .
$$

Combined (16) with Proposition 4, we can assert that there exists $c>0$ such that for $\psi \in H^{1 / 2}(\mathbb{R})$,

$$
\|\psi\|_{4}^{4} \leq c\|\psi\|_{2}^{2}\langle\psi, L \psi\rangle,
$$

therefore, from (17), the following quotient is bounded from below

$$
\mathcal{V}_{L}(\psi)=: \frac{\langle\psi, L \psi\rangle\|\psi\|_{2}^{2}}{\|\psi\|_{4}^{4}} \geq c .
$$

Now using (11) and (16), we have that there exists a positive constant

$$
v_{L}=: \inf _{\psi \in H^{1 / 2}(\mathbb{R})} \mathcal{V}_{L}(\psi)>0
$$

We can define the energy operator for $L$, such as in (2), as it follows:

$$
\mathcal{E}(\psi)=\frac{1}{2}\langle\psi, L \psi\rangle-\frac{\gamma}{4}\|\psi\|_{4}^{4} .
$$




\section{B. Local and global existence of solutions}

In this section, we prove local and global existence of solutions of the general pseudo-differential problem given in (15).

Notice that if $\psi(t, x)$ is a solution of $(15)$, then $\tilde{\psi}(t, x)=\psi^{*}(-t,-x)$ verifies

$$
\begin{aligned}
i \tilde{\psi}_{t} & =L \tilde{\psi}-\gamma|\tilde{\psi}|^{2} \tilde{\psi}, \\
\left.\tilde{\psi}\right|_{t=0} & =\tilde{\psi}_{0},
\end{aligned}
$$

where $\tilde{\psi}_{0}(x)=\psi_{0}^{*}(-x)$, then in order to solve (15) it is enough to consider only $t \geq 0$.

Theorem 6 (Local Existence): Let $s>1 / 2$ and $\psi_{0} \in H^{s}(\mathbb{R})$, then there exists $T^{*}$ $=T^{*}\left(\left\|\psi_{0}\right\|_{H^{s}}\right)>0$ such that for all $0<T<T^{*},(15)$ has a unique solution $\psi \in C\left([0, T], H^{s}(\mathbb{R})\right) \cap$ $C^{1}\left([0, T], H^{s-1}(\mathbb{R})\right)$. Also, application $\psi_{0} \mapsto \psi$ from $H^{s}(\mathbb{R})$ in $C\left([0, T], H^{s}(\mathbb{R})\right)$ is local Lipschitz continuous.

Proof: If $S(t)=\exp (-i t L), \psi$ is a solution of the problem (15) if and only if it verifies the fix point equation given by

$$
\psi(t)=S(t) \psi_{0}+i \gamma \int_{0}^{t} S\left(t-t^{\prime}\right)\left|\psi\left(t^{\prime}\right)\right|^{2} \psi\left(t^{\prime}\right) d t^{\prime} .
$$

Since $S$ is an unitary group in $H^{s}(\mathbb{R})$ and using (10) and the fix point Banach's theorem, conclusion follows.

Proposition 7 (Conservation Laws): Let $s>1 / 2$ and $\psi \in C\left(\left[0, T^{*}\right), H^{s}(\mathbb{R})\right)$ a solution of the problem (15), then $\mathcal{N}(\psi(t))=\mathcal{N}\left(\psi_{0}\right)$ and $\mathcal{E}(\psi(t))=\mathcal{E}\left(\psi_{0}\right)$ for all $t \in\left[0, T^{*}\right)$, where $\mathcal{E}(\psi(t))$ was given in (20).

Proof: If $s \geq 1$, we have that $\psi \in C^{1}\left([0, T], L^{2}(\mathbb{R})\right)$, then, from the continuous embedding of $H^{1}(\mathbb{R})$ into $L^{p}(\mathbb{R})$ and from the fact that $L$ is a bound operator from $H^{1 / 2}(\mathbb{R})$ in $H^{-1 / 2}(\mathbb{R})$, we have that $\mathcal{N}(\psi)$ and $\mathcal{E}(\psi)$ are differentiable operators on the time. Then

$$
\begin{aligned}
& \frac{d}{d t} \mathcal{N}(\psi(t))=2\left\langle\psi_{t}, \psi\right\rangle=2\left\langle-i L \psi+i \gamma|\psi|^{2} \psi, \psi\right\rangle=0, \\
& \frac{d}{d t} \mathcal{E}(\psi(t))=\left\langle\psi_{t}, L \psi-\gamma|\psi|^{2} \psi\right\rangle=\left\langle-i L \psi+i \gamma|\psi|^{2} \psi, L \psi-\gamma|\psi|^{2} \psi\right\rangle=0,
\end{aligned}
$$

therefore, $\mathcal{N}(\psi(t))$ and $\mathcal{E}(\psi(t))$ are constants.

If $1 / 2<s<1$, we take a sequence $\left\{\rho_{n}\right\}_{n \in \mathbb{N}} \subset H^{1}(\mathbb{R})$ such that it verifies $\rho_{n} \rightarrow \psi_{0}$ in $H^{s}(\mathbb{R})$. If $\psi_{n}$ is the solution of the problem (15) with initial data $\rho_{n}$, we know that $\psi_{n} \rightarrow \psi$ in $C\left([0, T], H^{s}(\mathbb{R})\right)$. But, due to $\mathcal{N}\left(\psi_{n}\right)$ and $\mathcal{E}\left(\psi_{n}\right)$ are constants and operators $\mathcal{N}$ and $\mathcal{E}$ are continuous in $H^{s}(\mathbb{R})$, conservation laws follow.

Corollary 8: If $\gamma \leq 0$, there exists a constant $C>0$ such that if $\psi \in C\left(\left[0, T^{*}\right), H^{s}(\mathbb{R})\right)$ is the solution of the problem (15) for $s>1 / 2$, then $\|\psi(t)\|_{H^{1 / 2}}^{2} \leq C \mathcal{E}\left(\psi_{0}\right)$ for all $t \in\left[0, T^{*}\right)$.

Proof: If $\gamma \leq 0$, we know that $\langle\psi, L \psi\rangle \leq \mathcal{E}(\psi)=\mathcal{E}\left(\psi_{0}\right)$, then the result follows from (16).

Lemma 9: Let $s>1 / 2$ and $\psi \in C\left(\left[0, T^{*}\right), H^{s}(\mathbb{R})\right)$ a solution of the problem (15), then

$$
\|\psi(t)\|_{H^{s}}^{2} \leq\left\|\psi_{0}\right\|_{H^{s}}^{2}+c_{s}|\gamma| \int_{0}^{t}\left\|\psi\left(t^{\prime}\right)\right\|_{\infty}^{2}\left\|\psi\left(t^{\prime}\right)\right\|_{H^{s}}^{2} d t^{\prime}
$$


Proof: If $\psi_{0} \in H^{s+1}(\mathbb{R})$, we know that $\|\psi\|_{H^{s}}^{2}$ is differentiable and by using (7), we have that

$$
\begin{aligned}
\frac{d}{d t}\|\psi(t)\|_{H^{s}}^{2}= & \left\langle J_{t}^{s} \psi, J^{s} \psi\right\rangle=\left\langle-i L J^{s} \psi, J^{s} \psi\right\rangle \\
& +\gamma\left\langle i J^{s}\left(|\psi|^{2} \psi\right), J^{s} \psi\right\rangle \leq c_{s}|\gamma|\|\psi\|_{\infty}^{2}\|\psi\|_{H^{s}}^{2},
\end{aligned}
$$

where, in the last estimation, we used Proposition 2 . Integrating in $[0, t]$, we obtain the inequality. The general case is proved by approximating $\varphi_{0}$ by functions of $H^{s+1}(\mathbb{R})$ and using the continuous dependence of the function $\psi_{0} \mapsto \psi$.

Corollary 10: Under above conditions, we have that

$$
\|\psi(t)\|_{H^{s}}^{2} \leq\left\|\psi_{0}\right\|_{H^{s}}^{2}+c_{s}|\gamma| \int_{0}^{t}\left(1+\left\|\psi\left(t^{\prime}\right)\right\|_{H^{1 / 2}}^{2} \log \left(2+\left\|\psi\left(t^{\prime}\right)\right\|_{H^{s}}\right)\right)\left\|\psi\left(t^{\prime}\right)\right\|_{H^{s}}^{2} d t^{\prime} .
$$

Lemma 11: Let $\psi_{0} \in H^{s}$ an initial data, $s>1 / 2$ and $\gamma>0$, then there exists $c>0$ such that if $\psi \in C\left(\left[0, T^{*}\right), H^{s}(\mathbb{R})\right)$ is a solution of the problem $(15)$ and $\mathcal{N}\left(\psi_{0}\right)<v_{L} / \gamma$, where $v$ is given in (19), then

$$
\|\psi(t)\|_{H^{1 / 2}}^{2} \leq c\left(1-\gamma / v_{L} \mathcal{N}\left(\psi_{0}\right)\right)^{-1} \mathcal{E}\left(\psi_{0}\right) \quad \text { for all } t \in\left[0, T^{*}\right)
$$

Proof: From (18) and (19), we have that

$$
\|\psi\|_{4}^{4} \leq v_{L}^{-1}\|\psi\|_{2}^{2}\langle\psi, L \psi\rangle=v_{L}^{-1}\left\|\psi_{0}\right\|_{2}^{2}\langle\psi, L \psi\rangle
$$

therefore, we have that

$$
\mathcal{E}\left(\psi_{0}\right)=\mathcal{E}(\psi) \geq \frac{1}{2}\left(1-\frac{\gamma}{v_{L}} \mathcal{N}\left(\psi_{0}\right)\right)\left\langle\psi_{0}, L \psi_{0}\right\rangle
$$

By using (16) and the above inequality, estimation (21) follows.

Now, we are in condition to deal with the global existence of solutions. We need to distinguish two cases, according to the sign of the parameter $\gamma$ at initial value problem (15).

Theorem 12 (Global Existence): Let $s>1 / 2$ and $\psi_{0} \in H^{s}$, we have that:

1. If $\gamma \leq 0$, there exists a unique $\psi \in C\left(\mathbb{R}, H^{s}(\mathbb{R})\right) \cap C^{1}\left(\mathbb{R}, H^{s-1}(\mathbb{R})\right)$ solution of the problem (15). In addition, if $T>0$, then function $\psi_{0} \mapsto \psi(t)$ is continuous from $H^{s}(\mathbb{R})$ in $C\left([-T, T], H^{s}(\mathbb{R})\right)$.

2. If $\gamma>0$ and $\mathcal{N}\left(\psi_{0}\right)<v_{L} / \gamma$, we have a similar conclusion like in the case $\gamma \leq 0$.

Proof: If $\gamma \leq 0$, we are going to prove that $T^{*}\left(\left\|\psi_{0}\right\|_{H^{s}}\right)=+\infty$. From Corollaries (8) and (10), we have that

$$
\|\psi(t)\|_{H^{s}}^{2} \leq\left\|\psi_{0}\right\|_{H^{s}}^{2}+c_{s}|\gamma| \int_{0}^{t}\left(1+\mathcal{E}\left(\psi_{0}\right) \log \left(2+\left\|\psi\left(t^{\prime}\right)\right\|_{H^{s}}\right)\right)\left\|\psi\left(t^{\prime}\right)\right\|_{H^{s}}^{2} d t^{\prime} .
$$

Let $A, B>0$ be, then function $f(\eta)=A(1+B \log (2+\sqrt{\eta})) \eta$ verifies the hypothesis of the Gronwall's lemma (5). Therefore, there exists $G \in C^{1}([0,+\infty))$ such that $\|\psi(t)\|_{H^{s}}^{2} \leq G(t)$. By an usual prolongation argument of solutions we can assert that $\psi(t)$ is defined on $[0,+\infty)$. The continuity on the initial data is a direct consequence of the local existence theorem.

If $\gamma>0$ and $\mathcal{N}\left(\psi_{0}\right)<v_{L} / \gamma$, conclusion follows from (21).

\section{STATIONARY PROBLEM}

\section{A. Basic estimates}

By using definitions given in (8) and the trivial inequality $|\xi| \leq \sqrt{m^{2}+\xi^{2}} \leq m+|\xi|$, we have the following relationship between their operators and also between their corresponding quadratic 
forms on $H^{1 / 2}(\mathbb{R})$,

$$
\begin{gathered}
J-m I \leq D \leq J, \\
\left\langle L_{m} \psi, \psi\right\rangle \leq\left\langle L_{0} \psi, \psi\right\rangle \leq\left\langle\left(L_{m}+m I\right) \psi, \psi\right\rangle,
\end{gathered}
$$

If $\varphi \in H^{1 / 2}(\mathbb{R})$ and $|v|<1$, taking note that inequality $\sqrt{m^{2}+\xi^{2}}-v \xi \geq m \sqrt{1-v^{2}}$ holds, and using the right side of $(9 \mathrm{~d})$, we have that

$$
\left\langle\varphi, L_{m} \varphi\right\rangle \geq-m\left(1-\sqrt{1-v^{2}}\right)\|\varphi\|_{2}^{2} .
$$

Lemma 13: If $\varphi \in H^{1 / 2}(\mathbb{R})$ and $|v|<1$, then

$$
\|\varphi\|_{4}^{4} \leq \frac{8}{\pi(1-|v|)}\left\langle\varphi, L_{0} \varphi\right\rangle\|\varphi\|_{2}^{2} .
$$

Proof: Since $|v|<1$, it is easy to check that $(1-|v|)|\xi| \leq|\xi|-v \xi \leq(1+|v|)|\xi|$. Using the above first inequality, we obtain

$$
\langle\varphi, D \varphi\rangle=\int_{R}|\xi||\hat{\varphi}|^{2} d \xi \leq \frac{1}{1-|v|} \int_{R}(|\xi|-v \xi)|\hat{\varphi}|^{2} d \xi=\frac{1}{1-|v|}\left\langle\varphi, L_{0} \varphi\right\rangle .
$$

Plugging (25) into (11), we obtain (24).

From now on we call $\mathcal{V}_{0}(\varphi)$ the quotient given in (18), where $L=L_{0}$.

As in (19), taking $L=L_{0}$, and using (24), we know that $\mathcal{V}_{0}$ is bounded from below and

$$
v_{0}(v)=\inf _{\varphi \in H^{1 / 2}(\mathbb{R})} \mathcal{V}_{0}(\varphi) \geq \frac{\pi(1-|v|)}{8} .
$$

In the next remark, we take note about the changes in the norm $L^{2}$, in the norm $L^{4}$ and in the quadratic form $(9 \mathrm{~b})$ when we introduce a rescaling.

Remark 14: Let $\varphi \in H^{1 / 2}(\mathbb{R}),|v|<1$ be, $y \in \mathbb{R}$ and $\mu, \lambda>0$, we consider a change of scale $\psi(x)=\mu^{1 / 2} \varphi(\lambda(x-y))$. It is easy to check that $\widehat{\psi}(\xi)=\mu^{1 / 2} \lambda^{-1} e^{-i y \xi} \widehat{\varphi}\left(\lambda^{-1} \xi\right)$, therefore,

$$
\begin{aligned}
& \|\psi\|_{2}^{2}=\mu \lambda^{-1}\|\varphi\|_{2}^{2}, \\
& \|\psi\|_{4}^{4}=\mu^{2} \lambda^{-1}\|\varphi\|_{4}^{4}, \\
& \left\langle\psi, L_{0} \psi\right\rangle=\mu \int_{\mathbb{R}}(|\xi|-v \xi) \lambda^{-2}\left|\hat{\varphi}\left(\lambda^{-1} \xi\right)\right|^{2} d \xi=\mu\left\langle\varphi, L_{0} \varphi\right\rangle,
\end{aligned}
$$

and

$$
\mathcal{V}_{0}(\psi)=\mathcal{V}_{0}(\varphi)
$$

The last part of this section is devoted to show how different norms change when we introduce a concentration function.

Proposition 15: Let $\rho \in C_{0}^{\infty}(\mathbb{R})$ given by

$$
\rho(x)=\left\{\begin{array}{ccc}
\exp \left(\frac{1}{x^{2}-1}+1\right) & \text { if } & |x|<1 \\
0 & \text { if } & |x| \geq 1
\end{array}\right.
$$


and its integer translations $\rho_{k}(x)=\rho(x-k)$, with $k \in \mathbb{Z}$. Then, there exists a constant $C>0$ such that, if $\varphi \in H^{1 / 2}(\mathbb{R})$, we have that

$$
\sum_{k \in \mathbb{N}}\left\|\rho_{k} \varphi\right\|_{H^{1 / 2}}^{2} \leq C\|\varphi\|_{H^{1 / 2}}^{2} .
$$

Proof: Notice that $\operatorname{supp}\left(\rho_{k}\right)=(k-1, k+1), 0 \leq \rho_{k}(x) \leq 1$, and $\left|\partial_{x} \rho_{k}(x)\right| \leq \frac{5}{2}$. Now, we make an inequality in the way of (29) for $\varphi \in L^{2}(\mathbb{R})$ and for $\varphi \in H^{1}(\mathbb{R})$. At last, we conclude (29) by an interpolation argument.

If $\varphi \in L^{2}(\mathbb{R})$, we have that

$$
\sum_{k \in \mathbb{Z}}\left\|\rho_{k} \varphi\right\|_{2}^{2} \leq 2\|\varphi\|_{2}^{2}
$$

If $\varphi \in H^{1}(\mathbb{R})$, we have the following bound:

$$
\sum_{k \in \mathbb{Z}}\left\|\partial_{x}\left(\rho_{k} \varphi\right)\right\|_{2}^{2} \leq \frac{35}{2}\|\varphi\|_{H^{1}}^{2} .
$$

From (30), we have that application $L^{2}(\mathbb{R}) \rightarrow l^{2}\left(\mathbb{Z}, L^{2}(\mathbb{R})\right)$, given by $\varphi \rightarrow\left(\rho_{k} \varphi\right)_{k}$, is continuous. Analogously, we have the same application $H^{1}(\mathbb{R}) \rightarrow l^{2}\left(\mathbb{Z}, H^{1}(\mathbb{R})\right)$. Then, by interpolation, we have that application $H^{1 / 2}(\mathbb{R}) \rightarrow l^{2}\left(\mathbb{Z}, H^{1 / 2}(\mathbb{R})\right)$ is continuous. Thus estimation (29) follows.

Lemma 16: Given $\rho(x)$ and $\rho_{k}(x)$ as to the lemma above, and $\varphi \in L^{4}(\mathbb{R})$, then

$$
\|\varphi\|_{4}^{4} \leq 2 \sum_{k \in \mathbb{Z}}\left\|\rho_{k} \varphi\right\|_{4}^{4} .
$$

Proof: For each $k \in \mathbb{Z}$, functions $\rho_{k}$ and $\rho_{k+1}$ are the only two functions of $\left\{\rho_{j}\right\}$ evaluated on the interval $[k, k+1]$. Moreover, it is easy to check that $\frac{1}{2} \leq \rho_{k}^{4}(x)+\rho_{k+1}^{4}(x) \leq 1$, and if $k-1$ $<x<k+1$, we have that $\frac{1}{2} \leq \rho_{k-1}^{4}(x)+\rho_{k}^{4}(x)+\rho_{k+1}^{4}(x) \leq 1$. Then inequality (32) follows.

Lemma 17: If $\varphi \in H^{1 / 2}(\mathbb{R})$ and $0 \leq|v|<1$, then

$$
\|\varphi\|_{4}^{4} \leq \frac{8}{\pi(1-|v|)} \sum_{k \in \mathbb{Z}}\left\|\rho_{k} \varphi\right\|_{2}^{2}\left\langle\rho_{k} \varphi, L_{0}\left(\rho_{k} \varphi\right)\right\rangle .
$$

Proof: Applying estimates (24) and (32), inequality (33) follows.

\section{B. Concentration-compactness principle and some of its consequences}

Now we present a concentration-compactness lemma adapted to our operator. This result is an adaptation of a similar one made in Ref. 5.

Lemma 18: Let $\left\{\varphi_{n}\right\}_{n \in \mathbb{N}}$ be a bounded sequence in $H^{1 / 2}(\mathbb{R})$ with $\mathcal{N}\left(\varphi_{n}\right)=N$, for all $n \geq 0$. Then there exists a subsequence $\left\{\varphi_{n_{k}}\right\}_{k \in \mathbb{N}}$ satisfying one of the three following properties:

1. Compactness: There exists a sequence $\left\{y_{k}\right\}_{k \in \mathbb{N}} \subset \mathbb{R}$ such that, for every $\epsilon>0$, there exists 0 $<R(\varepsilon)<\infty$ with

$$
\int_{\left|x-y_{k}\right| \leq R}\left|\varphi_{n_{k}}(x)\right|^{2} d x \geq N-\varepsilon .
$$

2. Vanishing: For all $R>0$,

$$
\lim _{k \rightarrow \infty} \sup _{y \in \mathbb{R}} \int_{|x-y| \leq R}\left|\varphi_{n_{k}}(x)\right|^{2} d x=0 .
$$


3. Dichotomy: There exists $\alpha \in(0, N)$ such that, for every $\varepsilon>0$, there exist $R>0,\left\{y_{k}\right\}_{k \in \mathbb{N}}$ $\subset \mathbb{R},\left\{R_{k}\right\}_{k \in \mathbb{N}} \subset(0, \infty)$, with $R_{k} \rightarrow \infty$, and two bounded sequences, $\left\{\psi_{k}^{1}\right\}_{k \in \mathbb{N}}$ and $\left\{\psi_{k}^{2}\right\}_{k \in \mathbb{N}} \subset$ $H^{1 / 2}(\mathbb{R})$, such that the following properties hold:

(a) $\operatorname{supp}\left(\psi_{k}^{1}\right) \subset\left(y_{k}-R, y_{k}+R\right), \quad \operatorname{supp}\left(\psi_{k}^{1}\right) \subset\left(y_{k}-R, y_{k}+R\right)^{c}$, as a consequence $\operatorname{dist}\left(\operatorname{supp}\left(\psi_{k}^{1}\right), \operatorname{supp}\left(\psi_{k}^{2}\right)\right) \rightarrow \infty$ as $k \rightarrow \infty$.

(b) $\left\|\varphi_{n_{k}}-\left(\psi_{k}^{1}+\psi_{k}^{2}\right)\right\|_{2}^{2} \leq \delta(\varepsilon)$ with $\delta(\varepsilon) \rightarrow 0$ as $\varepsilon \rightarrow 0$.

(c) $\left|\left\|\psi_{k}^{1}\right\|_{2}^{2}-\alpha\right|<\varepsilon \quad$ and $\left|\left\|\psi_{k}^{2}\right\|_{2}^{2}-(N-\alpha)\right|<\varepsilon$.

(d) For all $m \geq 0$ : $\lim _{k \rightarrow \infty} \inf \left(\left\langle\varphi_{n_{k}}, L_{m} \varphi_{n_{k}}\right\rangle-\left\langle\psi_{k}^{1}, L_{m} \psi_{k}^{1}\right\rangle-\left\langle\psi_{k}^{2}, L_{m} \psi_{k}^{2}\right\rangle\right) \geq-C(\varepsilon) \underset{\varepsilon \rightarrow 0}{\rightarrow} 0$.

Proof: See Ref. 5.

In the last part of this section, we present some consequences when each property of Lemma 18 is satisfied.

First, we show a consequence if compactness (property 1 in Lemma 18) is satisfied.

Lemma 19 (Local compactness): Let $A$ be a bounded set in $H^{1 / 2}(\mathbb{R})$, such that, for all $\varphi \in$ $A,\|\varphi\|_{2}=N$, and for any $\varepsilon>0$ there exists $R>0$ such that $\int_{-R}^{R}|\varphi|^{2} d x \geq N-\varepsilon, \forall \varphi \in A$, then $A$ is a relatively compact set of $L^{2}(-R, R)$.

Proof: It follows from the Frechet-Kolmogorov strong compactness principle in $L^{p}$ (see Ref. 3). It is enough to prove that for all $R>0$,

- $\forall \varphi \in A:\|\varphi\|_{L^{2}(\mathbb{R})} \leq C$.

- $\forall \varepsilon>0, \exists \delta(\varepsilon)>0$, such that $\forall|h|<\delta$ and $\forall \varphi \in A$, it satisfies $\left\|\tau_{h} \varphi-\varphi\right\|_{L^{2}(-R, R)}<\varepsilon$, where $\tau_{h} \varphi(x)=\varphi(x+h)$.

First condition is verified by hypothesis. In order to verify the second condition, we can calculate

$$
\left\|\tau_{h} \varphi-\varphi\right\|_{2}^{2}=\left\|\left(\tau_{h} \varphi-\varphi\right)^{\wedge}\right\|_{2}^{2}=\int_{\mathbb{R}}(2-2 \cos (h \xi))|\hat{\varphi}|^{2} d \xi .
$$

By hypothesis $\|\varphi\|_{H^{1 / 2}}^{2} \leq C$ and $g_{h}(x)=\frac{2-2 \cos (h x)}{\sqrt{1+x^{2}}} \leq \frac{8}{\pi} h$, then second condition holds.

Now, we present a result when vanishing (property 2 in Lemma 18) is satisfied.

Lemma 20: Let $\left\{\varphi_{n}\right\}_{n \in \mathbb{N}} \subset H^{1 / 2}(\mathbb{R})$ satisfying property 2 in Lemma 18, then the subsequence $\left\{\varphi_{n_{k}}\right\}$ given in that property, verifies

$$
\left\|\varphi_{n_{k}}\right\|_{4} \underset{k \rightarrow+\infty}{\rightarrow} 0
$$

Proof: Applying (33), with $v=0$, on each element of this subsequence, we have that

$$
\left\|\varphi_{n_{k}}\right\|_{4}^{4} \leq \frac{8}{\pi} \sum_{j \in \mathbb{Z}}\left\|\rho_{j} \varphi_{n_{k}}\right\|_{H^{1 / 2}}^{2}\left\|\rho_{j} \varphi_{n_{k}}\right\|_{2}^{2} \quad \forall k \in \mathbb{Z}
$$

By hypothesis $\left\|\rho_{j} \varphi_{n_{k}}\right\|_{2}^{2}<\varepsilon$ for $k \geq k_{0}$ uniformly in $j \in \mathbb{Z}$, then for (29) we have that

$$
\sum_{j \in \mathbb{Z}}\left\|\rho_{j} \varphi_{n_{k}}\right\|_{H^{1 / 2}}^{2} \leq C\left\|\varphi_{n_{k}}\right\|_{H^{1 / 2}}^{2} \leq C
$$

because subsequence $\left\{\varphi_{n_{k}}\right\}$ is bounded in $H^{1 / 2}(\mathbb{R})$. Thus

$$
\left\|\varphi_{n_{k}}\right\|_{4}^{4} \leq \frac{8}{\pi} C \varepsilon \underset{k \rightarrow+\infty}{\rightarrow} 0,
$$

which proves (34). 
At last, we show two results when dichotomy (property 3 in Lemma 18) is satisfied.

Lemma 21: Let $\left\{\varphi_{n}\right\}_{n \in \mathbb{N}} \subset H^{1 / 2}(\mathbb{R})$ satisfying property 3 in Lemma 18. If $\left\{\psi_{k}^{1}\right\}_{k \in \mathbb{N}},\left\{\psi_{k}^{2}\right\}_{k \in \mathbb{N}}$ $\subset H^{1 / 2}(\mathbb{R})$ are the subsequences given at the dichotomy property, we have that

$$
\lim _{k \rightarrow \infty}\left(\left\langle\psi_{k}^{1}+\psi_{k}^{2}, L_{0}\left(\psi_{k}^{1}+\psi_{k}^{2}\right)\right\rangle-\left\langle\psi_{k}^{1}, L_{0} \psi_{k}^{1}\right\rangle-\left\langle\psi_{k}^{2}, L_{0} \psi_{k}^{2}\right\rangle\right)=0
$$

Proof: From (3a) and using that $\partial_{x}$ is a local operator we have that

$$
\lim _{k \rightarrow \infty}\left\langle\psi_{k}^{2}, i v \partial_{x} \psi_{k}^{1}\right\rangle=\lim _{k \rightarrow \infty}\left\langle\psi_{k}^{1}, i v \partial_{x} \psi_{k}^{2}\right\rangle=0
$$

Then, in order to prove that limit (35) holds, it is enough to prove that

$$
\lim _{k \rightarrow \infty}\left\langle\psi_{k}^{2}, D \psi_{k}^{1}\right\rangle=\lim _{k \rightarrow \infty}\left\langle\psi_{k}^{1}, D \psi_{k}^{2}\right\rangle=0
$$

Due to our problem is invariant under translations, we can suppose that $y_{k}=0$, for all $k$. Let $\chi_{k} \in C_{0}^{\infty}(\mathbb{R})$ with $0 \leq \chi_{k} \leq 1, \chi_{k} \equiv 1$ in $(-R, R), \chi_{k} \equiv 0$ in $\left(-R_{k}, R_{k}\right)^{c}$ and such that $\left\|\partial_{x} \chi\right\|_{\infty} \leq$ $C /\left(R_{k}-R\right)$, then

$$
\left\langle\psi_{k}^{2}, D \psi_{k}^{1}\right\rangle=\left\langle\psi_{k}^{2}, D \chi_{k} \psi_{k}^{1}\right\rangle=\left\langle\psi_{k}^{2}, \chi_{k} D \psi_{k}^{1}\right\rangle+\left\langle\psi_{k}^{2},\left[D, \chi_{k}\right] \psi_{k}^{1}\right\rangle .
$$

From Lemma 183a, we have that $\left\langle\psi_{k}^{2}, \chi_{k} D \psi_{k}^{1}\right\rangle=0$, and by using Proposition 3 we have that $\left|\left\langle\psi_{k}^{2},\left[D, \chi_{k}\right] \psi_{k}^{1}\right\rangle\right| \leq C /\left(R_{k}-R\right)$, then (35) follows.

Lemma 22: Let $\left\{\varphi_{n}\right\}_{n \in \mathbb{N}} \subset H^{1 / 2}(\mathbb{R})$ be, satisfying property 3 in Lemma 18 , and if $\left\{\psi_{k}^{1}\right\}_{k \in \mathbb{N}}$, $\left\{\psi_{k}^{2}\right\}_{k \in \mathbb{N}} \subset H^{1 / 2}(\mathbb{R})$ are the subsequences given in the dichotomy property, then

$$
\left\|\varphi_{n_{k}}\right\|_{4}^{4} \leq\left\|\psi_{k}^{1}\right\|_{4}^{4}+\left\|\psi_{k}^{2}\right\|_{4}^{4}+o(\varepsilon) .
$$

Proof: We call $\beta_{k}=\varphi_{n_{k}}-\left(\psi_{k}^{1}+\psi_{k}^{2}\right)$ and from $\left\{\psi_{k}^{1}+\psi_{k}^{2}\right\}_{k \in \mathbb{N}}$ is bounded in $H^{1 / 2}(\mathbb{R})$. From (24), we have that

$$
\left\|\beta_{k}\right\|_{4}^{4}=\left\|\varphi_{n_{k}}-\left(\psi_{k}^{1}+\psi_{k}^{2}\right)\right\|_{4}^{4} \leq C\left\|\varphi_{n_{k}}-\left(\psi_{k}^{1}+\psi_{k}^{2}\right)\right\|_{2}^{2} \rightarrow 0 \quad \text { with } k \rightarrow+\infty
$$

In order to prove inequality (36), we can consider

$$
\left\|\varphi_{n_{k}}\right\|_{4}^{4}=\left\|\beta_{k}+\psi_{k}^{1}+\psi_{k}^{2}\right\|_{4}^{4}=\left\|\psi_{k}^{1}\right\|_{4}^{4}+\left\|\psi_{k}^{2}\right\|_{4}^{4}+\left\|\beta_{k}\right\|_{4}^{4}+\sum_{j=1}^{12} I_{j},
$$

where $I_{j}=c \int_{\mathbb{R}}\left|\psi_{k}^{1}\right|^{\alpha}\left|\psi_{k}^{2}\right|^{\gamma}\left|\beta_{k}\right|^{\delta} d x$ with $\alpha+\gamma+\delta=4$. By hypothesis $\psi_{k}^{1}$ and $\psi_{k}^{2}$ are bounded in $H^{1 / 2}(\mathbb{R})$ and $\operatorname{dist}\left(\operatorname{supp}\left(\psi_{k}^{1}\right), \operatorname{supp}\left(\psi_{k}^{2}\right)\right) \rightarrow 0$ when $k \rightarrow \infty$. Therefore, from (37) for all $1 \leq j \leq$ 12 , we have that $I_{j} \rightarrow 0$ when $k \rightarrow \infty$. Hence (36) follows.

\section{Existence of minimum of $\mathcal{V}_{0}$}

In this section, we prove that the infimum $v_{0}$ (see (26)) is reached on $H^{1 / 2}(\mathbb{R})$.

Proposition 23: Given $|v|<1$, there exists $Q_{v} \in H^{1 / 2}(\mathbb{R})$ such that $\mathcal{V}_{0}\left(Q_{v}\right)=v_{0}(v)\left(Q_{v}\right.$ is a minimum of $\left.\mathcal{V}_{0}\right)$. In addition $\left\langle Q_{v}, Q_{v}\right\rangle=2 v_{0}(v)$. Therefore,

$$
\left\|Q_{v}\right\|_{4}^{4}=\frac{1}{v_{0}}\left\langle Q_{v}, L_{0} Q_{v}\right\rangle\left\|Q_{v}\right\|_{2}^{2}=2\left\langle Q_{v}, L_{0} Q_{v}\right\rangle .
$$

Proof: Let $\left\{\varphi_{n}\right\}_{n \in \mathbb{N}} \subset H^{1 / 2}(\mathbb{R})$ a minimizer sequence of (26). In (27a) and (27c), we can take suitable values $\lambda$ and $\mu$ so that we can suppose $\left\|\varphi_{n}\right\|_{2}^{2}=\alpha$ and $\left\langle\varphi_{n}, L_{0} \varphi_{n}\right\rangle=\beta$, for prescribed $\alpha$ and $\beta \in \mathbb{R}$, such that this sequence satisfies the hypothesis of Lemma 18. Then, there exists a subsequence $\left\{\varphi_{n_{k}}\right\}_{k \in \mathbb{N}}$ such that satisfies one of the three possible alternatives. 
At a first step, we prove that vanishing or dichotomy hold we obtain respective contradictions, then, since Lemma 18, compactness happens.

Let us suppose that case 2 of Lemma 18 occurs. At the begin of this proof, we can take $\left\|\varphi_{n_{k}}\right\|_{2}^{2}=\alpha$ and $\left\langle\varphi_{n_{k}}, L_{0} \varphi_{n_{k}}\right\rangle=\beta$, then the minimization problem (26) is equivalent to the following maximization problem:

$$
\mathfrak{I}(\alpha, \beta)=\sup _{\varphi \neq 0}\left\{\|\varphi\|_{4}^{4} /\|\varphi\|_{2}^{2}=\alpha \text { and }\left\langle\varphi, L_{0} \varphi\right\rangle=\beta\right\} .
$$

Notice that in the particular case $\alpha=\beta=1$, from (24) we know that

$$
0<\mathfrak{I}(1,1) \leq \frac{8}{\pi(1-|v|)} .
$$

If $\left\{\varphi_{n}\right\}$ is a maximizer sequence, there exists a subsequence $\left\{\varphi_{n_{k}}\right\}$ such that $\frac{1}{2} \mathfrak{I}(1,1) \leq\left\|\varphi_{n_{k}}\right\|_{4}^{4}<$ $\mathfrak{I}(1,1)$. Due to this sequence satisfies 2 of Lemma 18, it verifies (34). Hence, $\left\|\varphi_{n_{k}}\right\|_{4}^{4}<\frac{1}{2} \mathfrak{I}(1,1)$ for all $k$ great enough, this is a contradiction.

Let us suppose that case 3 of Lemma 18 occurs. Let $\left\{\varphi_{n}\right\}$ be a maximizer sequence of $\mathfrak{I}(1,1)$ and $\left\{\varphi_{n_{k}}\right\}$ is a subsequence for which there exist the sequences $\left\{\psi_{k}^{1}\right\}$ and $\left\{\psi_{k}^{2}\right\}$ given in 3 of Lemma 18 . For $\varepsilon>0$, there exists $\alpha \in \mathbb{R}$ such that for $k$ greater enough, we have that

$$
\begin{gathered}
\left\|\varphi_{n_{k}}-\left(\psi_{k}^{1}+\psi_{k}^{2}\right)\right\|_{2}^{2}<\varepsilon, \\
\left|\left\|\psi_{k}^{1}\right\|_{2}^{2}-\alpha\right|<\varepsilon \text { and }\left|\left\|\psi_{k}^{2}\right\|_{2}^{2}-(1-\alpha)\right|<\varepsilon, \\
\left\langle\psi_{k}^{1}+\psi_{k}^{2}, L_{0}\left(\psi_{k}^{1}+\psi_{k}^{2}\right)\right\rangle \leq\left\langle\varphi_{n_{k}}, L_{0} \varphi_{n_{k}}\right\rangle+\varepsilon .
\end{gathered}
$$

We define the rescaled functions $\varphi_{k}^{1}(x)=\psi_{k}^{1}\left(\lambda_{1} x\right)$ and $\varphi_{k}^{2}(x)=\mu^{1 / 2} \psi_{k}^{2}\left(\lambda_{2} x\right)$. As in (27a)-(27c), we have that

$$
\begin{array}{ll}
\left\|\varphi_{k}^{1}\right\|_{2}^{2}=\lambda_{1}^{-1}\left\|\psi_{k}^{1}\right\|_{2}^{2}, & \left\|\varphi_{k}^{2}\right\|_{2}^{2}=\mu \lambda_{2}^{-1}\left\|\psi_{k}^{2}\right\|_{2}^{2}, \\
\left\|\varphi_{k}^{1}\right\|_{4}^{4}=\lambda_{1}^{-1}\left\|\psi_{k}^{1}\right\|_{4}^{4}, & \left\|\varphi_{k}^{2}\right\|_{4}^{4}=\mu^{2} \lambda_{2}^{-1}\left\|\psi_{k}^{2}\right\|_{4}^{4}, \\
\left\langle\varphi_{k}^{1}, L_{0} \varphi_{k}^{1}\right\rangle=\left\langle\psi_{k}^{1}, L_{0} \psi_{k}^{1}\right\rangle, & \left\langle\varphi_{k}^{2}, L_{0} \varphi_{k}^{2}\right\rangle=\mu\left\langle\psi_{k}^{2}, L_{0} \psi_{k}^{2}\right\rangle .
\end{array}
$$

If we call $\beta=\left\langle\psi_{k}^{1}, L_{0} \psi_{k}^{1}\right\rangle$, from (35) and $\left\langle\varphi_{n_{k}}, L_{0} \varphi_{n_{k}}\right\rangle=1$, it is easy to check that $\beta<1$. Fixing $\lambda_{1}$ $=\frac{\left\|\psi_{k}^{1}\right\|_{2}^{2}}{\alpha}, \lambda_{2}=\frac{(1-\beta)\left\|\psi_{k}^{2}\right\|_{2}^{2}}{(1-\alpha)\left\langle\psi_{k}^{2}, L_{0} \psi_{k}^{2}\right\rangle}$, and $\mu=\frac{1-\beta}{\left\langle\psi_{k}^{2}, L_{0} \psi_{k}^{2}\right\rangle}$ we have that $\left\|\varphi_{k}^{1}\right\|_{2}^{2}=\alpha,\left\langle\varphi_{k}^{1}, L_{0} \varphi_{k}^{1}\right\rangle=\beta,\left\|\varphi_{k}^{2}\right\|_{2}^{2}$ $=1-\alpha$, and $\left\langle\varphi_{k}^{2}, L_{0} \varphi_{k}^{2}\right\rangle=1-\beta$. Hence

$$
\left\|\varphi_{k}^{1}\right\|_{4}^{4} \leq \mathfrak{I}(\alpha, \beta) \quad \text { and } \quad\left\|\varphi_{k}^{2}\right\|_{4}^{4} \leq \mathfrak{I}(1-\alpha, 1-\beta)
$$

and, moreover,

$$
\begin{aligned}
\left\|\psi_{k}^{1}\right\|_{4}^{4} & =\frac{\left\|\psi_{k}^{1}\right\|_{2}^{2}}{\alpha}\left\|\varphi_{k}^{1}\right\|_{4}^{4}, \\
\left\|\psi_{k}^{2}\right\|_{4}^{4} & =\frac{\left\langle\psi_{k}^{2}, L_{0} \psi_{k}^{2}\right\rangle\left\|\psi_{k}^{2}\right\|_{2}^{2}}{(1-\alpha)(1-\beta)}\left\|\varphi_{k}^{2}\right\|_{4}^{4} .
\end{aligned}
$$

In order to prove the subadditivity property

$$
\mathfrak{I}(1,1) \leq \mathfrak{I}(\alpha, \beta)+\mathfrak{I}(1-\alpha, 1-\beta),
$$

we only need, from (43), to prove that $\left\|\varphi_{n_{k}}\right\|_{4}^{4} \leq\left\|\varphi_{k}^{1}\right\|_{4}^{4}+\left\|\varphi_{k}^{2}\right\|_{4}^{4}+o(\varepsilon)$.

From (36), we know

$$
\left\|\varphi_{n_{k}}\right\|_{4}^{4} \leq\left\|\psi_{k}^{1}\right\|_{4}^{4}+\left\|\psi_{k}^{2}\right\|_{4}^{4}+o(\varepsilon)
$$


now, by a simple calculus we have that

$$
\begin{aligned}
\left\|\psi_{k}^{1}\right\|_{4}^{4}+\left\|\psi_{k}^{2}\right\|_{4}^{4}= & \frac{\left(\left\|\psi_{k}^{1}\right\|_{2}^{2}-\alpha\right)}{\alpha}\left\|\varphi_{k}^{1}\right\|_{4}^{4}+\left\|\varphi_{k}^{1}\right\|_{4}^{4}+\frac{\left\langle\psi_{k}^{2}, L_{0} \psi_{k}^{2}\right\rangle\left(\left\|\psi_{k}^{2}\right\|_{2}^{2}-(1-\alpha)\right)}{(1-\alpha)(1-\beta)}\left\|\varphi_{k}^{2}\right\|_{4}^{4} \\
& +\frac{\left\langle\psi_{k}^{2}, L_{0} \psi_{k}^{2}\right\rangle}{1-\beta}\left\|\varphi_{k}^{2}\right\|_{4}^{4} .
\end{aligned}
$$

Using (24) and (41) in the first and third terms, we have that both terms are $\circ(\varepsilon)$. In addition, from (35) and (42), we have that $\frac{\left\langle\psi_{k}^{2}, L_{0} \psi_{k}^{2}\right\rangle}{1-\beta} \leq 1$. Then we conclude that

$$
\left\|\varphi_{n_{k}}\right\|_{4}^{4} \leq\left\|\varphi_{k}^{1}\right\|_{4}^{4}+\left\|\varphi_{k}^{2}\right\|_{4}^{4}+o(\varepsilon)
$$

then we proved inequality (44).

By rescaling, it is easy to check that

$$
\mathfrak{I}(\alpha, \beta)=\alpha \beta \mathfrak{I}(1,1)
$$

and plugging this expression into (44) we have that

$$
1 \leq \alpha \beta+(1-\alpha)(1-\beta)=1+2 \alpha \beta-\alpha-\beta,
$$

then

$$
0 \leq \alpha(\beta-1)+\beta(\alpha-1)
$$

this is a contradiction because $0<\alpha, \beta<1$.

This says neither alternatives 2 and 3 of Lemma 18 are true for a minimizer sequence $\left\{\varphi_{n}\right\}$, then this sequence satisfies alternative 1 of Lemma 18 (compactness).

We define $\phi_{k}(x)=\varphi\left(x-y_{k}\right)$, then $\left\{\phi_{k}\right\}$ is compact in $L^{2}(\mathbb{R})$ (see Lemma 19), hence there exists a subsequence, that without of generality we can call $\phi_{k}$, such that $\lim _{k \rightarrow \infty} \phi_{k}=\tilde{Q}_{v} \in L^{2}(\mathbb{R})$. Due to this sequence is bounded in $H^{1 / 2}(\mathbb{R})$, we can take a new subsequence such that it converges weakly to $\tilde{Q}_{v}$, i.e., $\phi_{k} \rightarrow \tilde{Q}_{v}$ in $H^{1 / 2}(\mathbb{R})$, therefore, $\left\langle\tilde{Q}_{v}, L_{0} \tilde{Q}_{v}\right\rangle \leq 1$. Using (24) we know that $\phi_{k}$ tends to $\tilde{Q}_{v}$ in $L^{4}(\mathbb{R})$, then $\mathcal{V}_{0}\left(\tilde{Q}_{v}\right) \leq v_{0}$. How $v_{0}$ is the infimum value of $\mathcal{V}_{0}$, then

$$
\mathcal{V}_{0}\left(\tilde{Q}_{v}\right)=v_{0}
$$

At last, we can consider $Q_{v}=\sqrt{2 v_{0}} \tilde{Q}_{v}$, then from (28) we have that $\mathcal{V}_{0}\left(\tilde{Q}_{v}\right)=\mathcal{V}_{0}\left(Q_{v}\right)$ and $\left\|Q_{v}\right\|_{2}^{2}=2 v_{0}$. Then (38) follows.

Definition 24: We note $N_{c}(v)=:\left\|Q_{v}\right\|_{2}^{2}$. Moreover, we know that $N_{c}(v)=2 v_{0}$.

\section{Existence of boosted ground states}

With the above results, especially Proposition 23, now we are able to follow a similar way as it was taken in Ref. 5. We prove the existence of solutions of Eq. (4) through finding the minimizers of the problem (5). Previously, we need to adapt some results to our case.

In a first step we remake, for our energy operator, the lemma where we set up $N_{c}$ as a critical value of the charge. Actually, $N_{c}$ is a critical value for $N$ because if $N<N_{c}$ there exists minimum and if $N>N_{c}$ it does not exist.

Lemma 25: Let $\varphi \in H^{1 / 2}(\mathbb{R})$ be, such that $N=\|\varphi\|_{2}^{2}$. If $m \geq 0$ and $|v|<1$, then

1. $\mathcal{E}_{v}(\varphi) \geq \frac{1}{2}\left(1-\frac{N}{N_{c}(v)}\right)\left\langle\varphi, L_{0} \varphi\right\rangle-\frac{m}{2} N$

2. If $E_{v}(N)$ is the minimum of the energy, given in (6), then

(a) If $0<N \leq N_{c}(v) \Rightarrow E_{v}(N) \geq-\frac{1}{2} m N$.

(b) If $N>N_{c}(v) \Rightarrow E_{v}(N)=-\infty$.

(c) If $0<N \leq N_{c}(v)$, all minimizer sequence is bounded. 
Proof: We prove each one of the above statements.

1. From (22b) and (38), we have that

$$
\mathcal{E}_{v}(\varphi) \geq \frac{1}{2}\left(1-\frac{N}{N_{c}(v)}\right)\left\langle\varphi, L_{0} \varphi\right\rangle-\frac{m}{2} N
$$

2. In order to prove that $N_{c}(v)$ is a critical value of the charge, we can consider the following cases:

(a) If $0<N \leq N_{c}(v)$, coefficient $\left(1-\frac{N}{N_{c}(v)}\right) \geq 0$. From (9b), it is easy to check that $\left\langle\varphi, L_{0} \varphi\right\rangle$ $\geq 0$, then using inequality (45) we have that

$$
E_{v}(N) \geq-\frac{m}{2} N
$$

(b) If $N>N_{c}(v)$, we consider the evaluation of the energy $\mathcal{E}_{v}$ on the straight line spanned by $Q_{v}$, and using that $\sqrt{m^{2}-\partial_{x}^{2}}-m \leq \sqrt{-\partial_{x}^{2}}$ (see (22a)),

$$
E_{v}(N) \leq \mathcal{E}_{v}\left(\lambda Q_{v}\right) \leq\left.\mathcal{E}_{v}\left(\lambda Q_{v}\right)\right|_{m=0} \quad \text { for all } \lambda \in \mathbb{R}
$$

The right side is explicitly computable using the definition of $\mathcal{E}_{v}$ and (38),

$$
\left.2 \mathcal{E}_{v}\left(\lambda Q_{v}\right)\right|_{m=0}=\lambda^{2}\left\langle Q_{v}, L_{0} Q_{v}\right\rangle-\frac{\lambda^{4}}{2}\left\|Q_{v}\right\|_{4}^{4}=\frac{\lambda^{2}-\lambda^{4}}{2}\left\|Q_{v}\right\|_{4}^{4} .
$$

Therefore,

$$
E_{v}(N) \leq\left.\mathcal{E}_{v}\left(\lambda Q_{v}\right)\right|_{m=0}=-\frac{\lambda^{2}\left(\lambda^{2}-1\right)}{4}\left\|Q_{v}\right\|_{4}^{4} .
$$

Taken $|\lambda|>1$, we have that $E_{v}(N) \leq \mathcal{E}_{v}\left(\lambda Q_{v}\right)<0$ and also $\mathcal{E}_{v}\left(\lambda Q_{v}\right) \underset{\lambda \rightarrow+\infty}{\rightarrow}-\infty$, then $E_{v}(N)=-\infty$.

(c) If $0<N<N_{c}(v)$, we know that for each $N$ the functional $\mathcal{E}_{v}(\varphi)$ has infimum. Fixed a value of $N$ we consider a minimizer sequence $\left\{\varphi_{n}\right\}_{n}$ such that $\left\|\varphi_{n}\right\|_{2}^{2}=N$. In order to prove that sequence remains bounded in $H^{1 / 2}(\mathbb{R})$ we only need to prove that term $\left\langle\varphi_{n}, D \varphi_{n}\right\rangle$ is bounded from above.

From inequalities (25) and (45), for each $\varphi_{n}$ we have that

$$
\mathcal{E}_{v}\left(\varphi_{n}\right) \geq C(1-|v|)\left\langle\varphi_{n}, D \varphi_{n}\right\rangle-\frac{m}{2} N
$$

but, due to $\varphi_{n}$ is a minimizer sequence, we have that $\left|\mathcal{E}_{v}\left(\varphi_{n}\right)\right| \leq M$ for $n$ greater enough, then $\left\langle\varphi_{n}, D \varphi_{n}\right\rangle \leq C$.

\section{Non-relativistic boundedness}

Next step is to provide an upper bound for $E_{v}(N)$ given for the energy of the non-relativistic ground state. At first term, we will define the non-relativistic problem and its energy.

Definition 26: Let $m>0$ and $|v|<1$, we define the following energy functional in $H^{1}(\mathbb{R})$ :

$$
\mathcal{E}_{v}^{n r}(\psi)=: \frac{\sqrt{1-v^{2}}}{4 m}\left\|\partial_{x} \psi\right\|_{2}^{2}-\frac{1}{4}\|\psi\|_{4}^{4}
$$

and its corresponding minimization problem

$$
E_{v}^{n r}(N)=: \inf \left\{\mathcal{E}_{v}^{n r}(\psi) / \psi \in H^{1}(\mathbb{R}), \mathcal{N}(\psi)=N\right\}
$$

with Euler-Lagrange equation

$$
-\frac{\sqrt{1-v^{2}}}{4 m} \partial_{x}^{2} \psi-|\psi|^{2} \psi=-\mu \psi
$$


The study of the stationary 1D cubic Schrödinger equation (48) is a classical subject in the literature, and it is very important for its physical means, although, actually, it is just a special case of the nonlinear potential-type equation. Existence of solution for this stationary equation was studied for many authors. For instance, existence in $\mathbb{R}$ was studied in Refs. 10 and 11 and the Cauchy Periodic problem, in an interval, in Ref. 2.

In order to give a bound of $E_{v}(N)$ using $E_{v}^{n r}(N)$, we need some previous results.

Lemma 27: Let $\psi \in H^{1}(\mathbb{R})$ an even function, with $\|\psi\|^{2}=N$. Consider $|v|<1$ and the one parametric family of functions

$$
\psi_{\lambda}(x):=e^{i \lambda v x} \psi(x), \quad \text { where } \lambda>0,
$$

then the following formulas hold:

$$
\begin{aligned}
\frac{i}{2}\left\langle\psi_{\lambda}, v \partial_{x} \psi_{\lambda}\right\rangle & =-\frac{\lambda|v|^{2}}{2} N, \\
\left\langle\psi_{\lambda},-\partial_{x}^{2} \psi_{\lambda}\right\rangle & =\left\langle\psi,-\partial_{x}^{2} \psi\right\rangle+\lambda^{2}|v|^{2} N .
\end{aligned}
$$

Proof: Equality (50) follows from the fact that $\psi(x)$ and $\hat{\psi}(\xi)$ are even functions. Equality (51) follows from $\operatorname{Re} \int_{\mathbb{R}}\left(\psi \partial_{x} \psi\right) d x=0$.

Lemma 28: If $\lambda>0$, the following inequality holds:

$$
\sqrt{m^{2}-\partial_{x}^{2}} \leq \frac{1}{2 \lambda}\left(-\partial_{x}^{2}+m^{2}+\lambda^{2}\right)
$$

Proof: Let $\varphi \in H^{1 / 2}(\mathbb{R})$, using Parseval's equality we have that

$$
\frac{1}{2 \lambda}\left\langle\varphi,\left(-\partial_{x}^{2}+m^{2}+\lambda^{2}\right) \varphi\right\rangle=\frac{1}{2 \lambda} \int_{\mathbb{R}}\left(\xi^{2}+m^{2}+\lambda^{2}\right)|\hat{\varphi}|^{2} d x
$$

and (52) follows.

Now, we are able to prove an upper bound for $E_{v}(N)$ using the energy of the non-relativistic ground state.

Proposition 29: Given $m>0$ and $|v|<1$, the following bound of the energy holds

$$
E_{v}(N) \leq E_{v}^{n r}(N)-\frac{1}{2}\left(1-\sqrt{1-v^{2}}\right) m N
$$

and, immediately, $E_{v}(N)<0$.

Proof: This proposition performs a similar result to it was proved in Ref. 5. In that work, the authors show an estimation similar to (53), working over the linear part of the energy. Actually, our problem differs from the problem discussed in Ref. 5 only in its nonlinear part, then this proposition is verified by a similar way. We are going to make a sketch of the proof that it was given there, adapted to our problem.

Let $\psi$ be an even function in $H^{1}(\mathbb{R})$, such that $\|\psi\|_{2}^{2}=N$, and $\psi_{\lambda}$ is like in (49). We look for a bound of $\mathcal{E}_{v}\left(\psi_{\lambda}\right)$ depending on $\lambda$, and then we prove the existence of $\lambda^{*}$, a minimizer of this bound.

By using (50)-(51), and $\left|\psi_{\lambda}\right|=|\psi|$, we have that

$$
\mathcal{E}_{v}\left(\psi_{\lambda}\right) \leq \frac{N}{4}\left(1-v^{2}\right) \lambda+\frac{\left\langle\psi,-\partial_{x}^{2} \psi\right\rangle+m^{2} N}{4 \lambda}-\left(\frac{1}{2} m N+\frac{1}{4}\|\psi\|_{4}^{4}\right) .
$$

Evaluating (54) at $\lambda^{*}=\frac{m}{\sqrt{1-v^{2}}}$, we have that

$$
E_{v}(N) \leq \mathcal{E}_{v}\left(\psi_{\lambda^{*}}\right) \leq-\frac{1}{2}\left(1-\sqrt{1-v^{2}}\right) m N+\mathcal{E}_{v}^{n r}(\psi) \quad \text { with } \psi \in H^{1}(\mathbb{R}) \text { even. }
$$


We know (see Ref. 10) that there exists an even $\psi_{*} \in H^{1}(\mathbb{R})$, where functional $\mathcal{E}_{v}^{n r}(\psi)$ reaches its minimum value, i.e., $E_{v}^{n r}(N)=\mathcal{E}_{v}^{n r}\left(\psi_{*}\right)$. Now, evaluating inequality (55) at $\psi_{*}$ we get estimation (53).

Due to $E_{v}^{n r}(N)<0$ and inequality (53), we have that $E_{v}(N)<0$.

\section{Subadditivity of the energy $E_{v}(N)$}

Now, we give a last previous result that we will use in the proof of the existence of ground states. It is a strict subadditivity condition by a general function.

Lemma 30: If $f: \mathbb{R} \rightarrow \mathbb{R}$ is a function such that $\forall x \in \mathbb{R}$ and $\forall \lambda>1: f(\lambda x)<\lambda f(x)$, then $f(x)$ satisfies the following strict subadditivity condition: given $x \in \mathbb{R}$ and $0<\alpha<|x|$, then

$$
f(x)<f(\alpha)+f(x-\alpha) .
$$

Proof: It is an elementary calculus.

In the following proposition, we use the above lemma y order to prove a strict subadditivity condition for the energy $E_{v}(N)$.

Proposition 31: If $m>0$ and $|v|<1$, then $E_{v}(N)$ satisfies the following strict subadditivity condition for $0<N<N_{c}(v)$ : if $0<\alpha<N$, then

$$
E_{v}(N)<E_{v}(\alpha)+E_{v}(N-\alpha) .
$$

Moreover, $E_{v}(N)$ is a strict decreasing and concave function on the interval $0<N<N_{c}(v)$.

Proof: Despite the differences between the nonlinear part of our model and the same term of the equation of Lemma 2.3 in Ref. 5, the technic of proving and the conclusion remains true. Now, we provide a sketch of the proof.

We take in account that from the definition of the non-relativistic energy, we know that $E_{v}^{n r}(N)$ $\leq E_{v=0}^{n r}(N)<0$, Using this estimation into inequality (53), we have

$$
E_{v}(N)<-\frac{1}{2}\left(1-\sqrt{1-v^{2}}\right) m N .
$$

Let $e_{v}(N):=\inf _{\|\varphi\|_{2}^{2}=1}\left\{\frac{1}{2}\left\langle\varphi, L_{m} \varphi\right\rangle-\frac{N}{4}\|\varphi\|_{4}^{4}\right\}$. It is easy to check that $e_{v}(N)$ is decreasing on $N$ and that $e_{v}(N)<0$. This follows from

$$
E_{v}(N)=N e_{v}(N)
$$

Moreover, $e_{v}(N)$ is strict decreasing on $N$. For proving this last assertion, it is enough to check that each $N>0$, there exists a constant $c_{N}>0$ such that

$$
\left\|\varphi_{n}\right\|_{4}^{4} \geq c_{N}>0 \quad \forall n
$$

This follows by making a proof by contradiction, as it was made in Ref. 5: supposing that any minimizer sequence $\left\{\varphi_{n}\right\}$ of $e_{v}(N)$ satisfies $\left\|\varphi_{n}\right\|_{4}^{4} \rightarrow 0$, then equality (59) and estimation (23) lead to a contradiction with (58).

Now, from equality (59) and the strict decrease of $e_{v}(N)$, we have that for all $\lambda>1$ and $0<N$ $<N_{c}$,

$$
E_{v}(\lambda N)<\lambda E_{v}(N)
$$

Then, function $E_{v}(N)$ satisfies the hypothesis of Lemma 30, then inequality (57) follows. 


\section{E. Main result}

Theorem 32: Given $m>0$ and $|v|<1$, let $\mathcal{E}_{v}(\varphi)$ and $E_{v}(N)$ as in (5) and (6), respectively. If $N_{c}(v)$ is the positive constant given in Definition 24, then the following hold:

1. For $0<N<N_{c}(v)$ problem (6) has a minimizer, $\varphi_{v} \in H^{1 / 2}(\mathbb{R})$, and it is a solution of Eq. (4) for some $\mu \in \mathbb{R}$. Moreover, every minimizer sequence $\left\{\varphi_{n}\right\}$ of problem (6) is relatively compact in $H^{1 / 2}(\mathbb{R})$ up to translations, i.e., there exists a sequence $\left\{y_{k}\right\} \subset \mathbb{R}$ and a subsequence $\left\{\varphi_{n_{k}}\right\}$ such that $\varphi_{n_{k}}\left(.+y_{k}\right) \rightarrow \varphi_{v}$ strongly in $H^{1 / 2}(\mathbb{R})$.

2. For $N \geq N_{c}(v)$, no minimizer exists for problem (6), even though $E_{v}(N)=-\frac{1}{2} m N$ in the case $N=N_{c}(v)$.

Proof Part 1: If $0<N<N_{c}(v)$, there exists a minimizer $\varphi_{v} \in H^{1 / 2}(\mathbb{R})$.

Let $\left\{\varphi_{n}\right\} \subset H^{1 / 2}(\mathbb{R})$, a minimizer sequence for problem (6), i.e., $\lim _{n \rightarrow \infty} \mathcal{E}_{v}\left(\varphi_{n}\right)=E_{v}(N)$ and $\left\|\varphi_{n}\right\|_{2}^{2}=N$. From Lemma 25 we know that, in this case, $E_{v}(N)>-\infty$ and a minimizer sequence is bounded in $H^{1 / 2}(\mathbb{R})$, then we can apply the concentration-compactness principle (Lemma 18). We need to prove that a minimizer sequence $\left\{\varphi_{n}\right\}$ does not satisfy neither vanishing nor dichotomy property.

1. Let us suppose that a minimizer sequence $\left\{\varphi_{n}\right\}$ satisfies property 2 in Lemma 18 (vanishing), then there exists a subsequence such that $\left\|\varphi_{n_{k}}\right\|_{4}^{4} \underset{k \rightarrow+\infty}{\rightarrow} 0$ (see (34)), but it is a contradiction (see the proof of inequality (60).

2. Let us suppose that a minimizer sequence $\left\{\varphi_{n}\right\}$ satisfies property 3 in Lemma 18 (dichotomy), and suppose that subsequence $\left\{\varphi_{n_{k}}\right\}$ and sequences $\left\{\psi_{k}^{1}\right\}$ and $\left\{\psi_{k}^{2}\right\}$ satisfy property 3 in Lemma 18. Therefore, from Lemma (18 $3 \mathrm{c}$ ), we know there exists $0<\alpha<N$ such that for all $\varepsilon>0$ and $k$ greater enough

$$
\left\|\psi_{k}^{1}\right\|_{2}^{2} \leq \alpha+\varepsilon \text { and }\left\|\psi_{k}^{2}\right\|_{2}^{2} \leq(N-\alpha)+\varepsilon .
$$

Using (36) joint to Lemma (18 $3 \mathrm{~d}$ ), we have that

$$
E_{v}(N)=\lim _{k \rightarrow \infty} \mathcal{E}_{v}\left(\psi_{n_{k}}\right) \geq \lim _{k \rightarrow \infty} \inf \mathcal{E}_{v}\left(\psi_{k}^{1}\right)+\lim _{k \rightarrow \infty} \inf \mathcal{E}_{v}\left(\psi_{k}^{2}\right)-o(\varepsilon)
$$

and due to $E_{v}$ decreases in $N$ and inequalities (62), we have that

$$
E_{v}(N) \geq E_{v}(\alpha+\varepsilon)+E_{v}(N-\alpha+\varepsilon)-r(\varepsilon) .
$$

Here, $E_{v}$ is continuous because it is strictly concave, then taking limit with $\varepsilon \rightarrow 0$,

$$
E_{v}(N) \geq E_{v}(\alpha)+E_{v}(N-\alpha)
$$

but this is a contradiction by the subadditivity of $E_{v}$ (see (57)).

In consequence, from Lemma 18, a minimizer sequence $\left\{\varphi_{n}\right\}$ satisfies the property of compactness, then it has a weak limit $\varphi_{v} \in H^{1 / 2}(\mathbb{R})$, up to translations. As a second step, we will prove that this sequence has a subsequence $\left\{\varphi_{n_{k}}\right\}$ such that $\varphi_{n_{k}} \rightarrow \varphi_{v}$ in $H^{1 / 2}(\mathbb{R})$ (strong convergence).

Due to $\left\{\varphi_{n_{k}}\right\}$ is bounded in $L^{2}(\mathbb{R})$, then it converges weakly to $\varphi_{v}$ in $L^{2}(\mathbb{R})$. Hence we can apply Theorem 8.6 of Ref. 7: weak convergence implies strong convergence over small sets, adapted to $H^{1 / 2}(\mathbb{R})$, i.e., $\varphi_{n_{k}} \rightarrow \varphi_{v}$ in $L^{p}$ for all $2 \leq p<\infty$, over compact sets. Due to $\left\{\varphi_{n_{k}}\right\}$ satisfies alternative 1 at Lemma 18, we know that the last condition hold, then

$$
\varphi_{n_{k}} \rightarrow \varphi_{v} \text { in } L^{p}(\mathbb{R}) \text { for all } 2 \leq p<\infty .
$$

Now, we will prove that the minimum value of $\mathcal{E}_{v}$ is reached at $\varphi_{v}$, i.e., $\mathcal{E}_{v}\left(\varphi_{v}\right)=E_{v}(N)$. For this is enough to prove that $\left\|\varphi_{n_{k}}\right\|_{4}^{4} \rightarrow\left\|\varphi_{v}\right\|_{4}^{4}$. Using (63) we have that

$$
\begin{aligned}
\left|\left\|\varphi_{n_{k}}\right\|_{4}^{4}-\left\|\varphi_{v}\right\|_{4}^{4}\right| & \leq\left(\int_{\mathbb{R}}\left(\varphi_{n_{k}}^{2}+\varphi_{v}^{2}\right)^{2}\left(\varphi_{n_{k}}+\varphi_{v}\right)^{2} d x\right)^{1 / 2}\left(\int_{\mathbb{R}}\left|\varphi_{n_{k}}-\varphi_{v}\right|^{2} d x\right)^{1 / 2} \\
& \leq C\left\|\varphi_{n_{k}}-\varphi_{v}\right\|_{2} .
\end{aligned}
$$


Hence, by using (63) with $p=2$ we obtain that

$$
\left\|\varphi_{n_{k}}\right\|_{4}^{4} \rightarrow\left\|\varphi_{v}\right\|_{4}^{4} .
$$

From Lemma A.4 of Ref. 5, we know the functional $L_{m}$ is weakly lower semi-continuous and, due to, $\varphi_{n_{k}} \rightarrow \varphi_{v}$ in $H^{1 / 2}(\mathbb{R})$ we have that

$$
\lim _{k \rightarrow \infty} \inf \left(L_{m} \varphi_{n_{k}}\right) \geq L_{m}\left(\varphi_{v}\right)
$$

then

$$
E_{v}(N)=\lim _{k \rightarrow \infty} \mathcal{E}_{v}\left(\varphi_{n_{k}}\right) \geq \mathcal{E}_{v}\left(\varphi_{v}\right) \geq E_{v}(N)
$$

and in consequence we have the equality $\mathcal{E}_{v}\left(\varphi_{v}\right)=E_{v}(N)$, then $\varphi_{v} \in H^{1 / 2}(\mathbb{R})$ is a minimum of the energy under restriction $\mathcal{N}\left(\varphi_{v}\right)=N$.

At last, in order to prove the strong convergence $\varphi_{n_{k}} \rightarrow \varphi_{v}$ in $H^{1 / 2}(\mathbb{R})$, we take note, again, of Lemma A.4 of Ref. 5, where was proved that is enough to see that $\lim _{k \rightarrow \infty} L_{m} \varphi_{n_{k}}=L_{m}\left(\varphi_{v}\right)$ in order to prove that $\varphi_{n_{k}} \rightarrow \varphi_{v}$ in $H^{1 / 2}(\mathbb{R})$. From (64) and the equality (65), we can assert this fact holds. Then

$$
\varphi_{n_{k}} \rightarrow \varphi_{v} \text { strongly in } H^{1 / 2}(\mathbb{R}) .
$$

Part 2: No minimizer exists for $N \geq N_{c}(v)$.

If $N>N_{c}(v)$, from Lemma $252(\mathrm{~b})$, we know that $E_{v}(N)=-\infty$, then no minimizer exists.

Now, we deal with the case $N=N_{c}(v)$. First, we prove the that $E_{v}(N)=-\frac{1}{2} m N$. From Lemma 252 (a) we know that $E_{v}(N) \geq-\frac{1}{2} m N$, then in order to prove the equality is enough to prove the opposite inequality. We take an optimizer $Q_{v} \in H^{1 / 2}(\mathbb{R})$, as it was given in (38), and we consider the rescaling $Q_{v}^{(\lambda)}(x)=\lambda^{1 / 2} Q_{v}(\lambda x)$, therefore $\left\|Q_{v}^{(\lambda)}\right\|_{2}^{2}=\left\|Q_{v}\right\|_{2}^{2}=N_{c}(v)$, then

$$
E_{v}(N) \leq \mathcal{E}_{v}\left(Q_{v}^{(\lambda)}\right)=\frac{1}{2} \int_{\mathbb{R}}\left(\sqrt{\lambda^{2} \xi^{2}+m^{2}}-\lambda|\xi|\right)\left|\hat{Q}_{v}(\xi)\right|^{2} d \xi-\frac{1}{2} m N_{c}(v) \underset{\lambda \rightarrow \infty}{\rightarrow}-\frac{1}{2} m N_{c}(v) .
$$

Finally, we prove that there does not exist a minimizer for problem (6) with $N=N_{c}(v)$. We argue by contradiction as follows. Suppose that $\tilde{\varphi}_{v} \in H^{1 / 2}(\mathbb{R})$ is a minimizer for (6), thus, from the previous result and the inequality $\sqrt{m^{2}-\partial_{x}^{2}}>\sqrt{-\partial_{x}^{2}}$, we have that

$$
\begin{aligned}
-\frac{1}{2} m N_{c}(v) & =\mathcal{E}_{v}\left(\tilde{\varphi}_{v}\right)=\frac{1}{2}\left\langle\tilde{\varphi}_{v}, L_{m} \tilde{\varphi}_{v}\right\rangle-\frac{1}{4}\left\|\tilde{\varphi}_{v}\right\|_{4}^{4} \\
& >\frac{1}{2}\left\langle\tilde{\varphi}_{v}, L_{0} \tilde{\varphi}_{v}\right\rangle-\frac{1}{2} m N_{c}(v)-\frac{1}{4}\left\|\tilde{\varphi}_{v}\right\|_{4}^{4},
\end{aligned}
$$

then

$$
\left\|\tilde{\varphi}_{v}\right\|_{4}^{4}>2\left\langle\tilde{\varphi}_{v}, L_{0} \tilde{\varphi}_{v}\right\rangle .
$$

But this is a contradiction because, using Definition 24 and Proposition 23,

$$
\frac{N_{c}(v)}{2}=v_{0} \leq \frac{\left\langle\tilde{\varphi}_{v}, L_{0} \tilde{\varphi}_{v}\right\rangle\left\|\tilde{\varphi}_{v}\right\|_{2}^{2}}{\left\|\tilde{\varphi}_{v}\right\|_{4}^{4}}
$$

then

$$
\left\|\tilde{\varphi}_{v}\right\|_{4}^{4} \leq 2\left\langle\tilde{\varphi}_{v}, L_{0} \tilde{\varphi}_{v}\right\rangle
$$

Hence, conclusion follows.

\section{REGULARITY AND WEAKLY ORBITAL STABILITY}

At the last part, we conclude with two very important properties of the boosted ground states solutions (3). The first property is the regularity of the ground states. 
Proposition 33: If $\varphi \in H^{1 / 2}(\mathbb{R})$ is a solution of $(4)$, then $\varphi \in H^{s}(\mathbb{R})$ for all $s \geq 1 / 2$.

Proof: We use a bootstrapping argument. We know that $H^{1 / 2}(\mathbb{R}) \hookrightarrow L^{p}(\mathbb{R})$ for all $2 \leq p$ $<\infty$, in particular $\varphi \in L^{6}(\mathbb{R})$ and therefore $|\varphi|^{2} \varphi \in L^{2}(\mathbb{R})$. Thus, a solution $\varphi$ of (4) satisfies $\left(\sqrt{-\partial_{x}^{2}+m^{2}}+i v \partial_{x}\right) \varphi \in L^{2}(\mathbb{R})$, and this implies $\varphi \in H^{1}(\mathbb{R})$. By repeating this argument we have that $\varphi \in H^{2}(\mathbb{R})$, and so on.

At the second term, we prove that solutions of Eq. (1) are weakly orbital stable, i.e., if the initial data $\psi_{0}(x) \in H^{s}(\mathbb{R})$, with $s>1 / 2$, is near to a ground state profile $\varphi_{v}$, in the sense of $H^{1 / 2}$ norm, thus the flux of Eq. (1) remains near to the set of ground states profiles, also in the sense of $H^{1 / 2}$ norm. More precisely, let $S_{v, N}$ be the set of minimizers of $\mathcal{E}_{v}$ with $\mathcal{N}\left(\varphi_{v}\right)=N$, i.e.,

$$
S_{v, N}=\left\{\varphi_{v} \in H^{1 / 2}(\mathbb{R}) / \mathcal{E}_{v}\left(\varphi_{v}\right)=E_{v}(N), \mathcal{N}\left(\varphi_{v}\right)=N\right\}
$$

Notice that, from Proposition 33, $\varphi_{v} \in S_{v, N}$ implies that $\varphi_{v} \in H^{s}(\mathbb{R})$ for all $s \geq 1 / 2$. Also, from Theorem 32, set $S_{v, N}$ is non-empty, for $N<N_{c}(v)$.

We define the following concept of stability.

Definition 34: The flux of Eq. (1) is weakly orbitally stable with regard to the set $S_{v, N}$ if for $\psi_{0}(x) \in H^{s}(\mathbb{R})$, with $s>1 / 2$, and every $\varepsilon>0$, there exists $\delta>0$ such that

$$
\inf _{\varphi_{v} \in S_{v, N}}\left\|\psi_{0}-\varphi_{v}\right\|_{H^{1 / 2}}<\delta \Rightarrow \sup _{t \geq 0} \inf _{\varphi_{v} \in S_{v, N}}\left\|\psi(t)-\varphi_{v}\right\|_{H^{1 / 2}}<\varepsilon
$$

where $\psi(t)=\psi(t, x)$ is the solution, at time $t$, of Eq. (1) with initial data $\psi_{0}$.

Theorem 35: Given $m>0,|v|<1,0<N<N_{c}(v)$ and initial data $\psi_{0} \in H^{s}$, with $s>1 / 2$, then the flux of Eq. (1) is weakly orbitally stable with regard to the set $S_{v, N}$.

Proof: We can work in the lines of Theorem 2 of Ref. 5. First, if $0<N<N_{c}(v) \leq N_{c}$, we can choose $\delta>0$ small enough such that if $\psi_{0}(x) \in H^{s}(\mathbb{R})$ and $\inf _{\varphi_{v} \in S_{v, N}}\left\|\psi_{0}-\varphi_{v}\right\|_{H^{1 / 2}}<\delta$ guarantees that $\mathcal{N}\left(\psi_{0}\right)<N_{c}$, then by the global well-posedness result for Eq. (1) given in Theorem 12, we have that its corresponding solution has global existence, thus taking sup $\operatorname{p}_{t \geq 0}$ is well defined.

Now, we assume that the weak orbital stability does not hold, then there exists $\varepsilon>0$ and a sequence of initial data $\left\{\psi_{n}(0)\right\}_{n} \subset H^{s}(\mathbb{R})$ such that

$$
\inf _{\varphi_{v} \in S_{v, N}}\left\|\psi_{n}(0)-\varphi_{v}\right\|_{H^{s}} \underset{n \rightarrow \infty}{\rightarrow} 0 \quad \text { and } \quad \inf _{\varphi_{v} \in S_{v, N}}\left\|\psi_{n}\left(t_{n}\right)-\varphi_{v}\right\|_{H^{s}}>\varepsilon \quad \text { for all } n \geq 0,
$$

where $t_{n}>0$ is a suitable sequence of times. Notice that from (66) and for the imbedding of $H^{s}(\mathbb{R})$ in $L^{2}(\mathbb{R})$, we have that $\mathcal{N}\left(\psi_{n}(0)\right) \rightarrow N$ as $n \rightarrow \infty$. Then, we can assume that $\mathcal{N}\left(\psi_{n}(0)\right)<N_{c}$ for all $n \geq 0$, which guarantees that the corresponding solution $\psi_{n}(t)$, exists globally in time.

From Proposition $7, \mathcal{N}(\psi)$ and $\mathcal{E}(\psi)$ are conserved by the flux of (1), and it is easy to check that the charge current $\left\langle i \partial_{x} \psi, \psi\right\rangle$ is also conserved, then we conclude that the energy $\mathcal{E}_{v}(\psi)$ is conserved.

We call $\phi_{n}(x):=\psi_{n}\left(x, t_{n}\right) \in H^{s}(\mathbb{R})$. By the conservation law of $\mathcal{N}(\psi)$ and $\mathcal{E}_{v}(\psi)$, we have that $\mathcal{N}\left(\phi_{n}\right)=\mathcal{N}\left(\psi_{n}(0)\right) \rightarrow N \quad$ as $n \rightarrow \infty$, and $\mathcal{E}_{v}\left(\phi_{n}\right)=\mathcal{E}_{v}\left(\psi_{n}(0)\right)$. Hence

$$
\lim _{n \rightarrow \infty} \mathcal{E}_{v}\left(\phi_{n}\right)=E_{v}(N) \text { and } \lim _{n \rightarrow \infty} \mathcal{N}\left(\phi_{n}\right)=N .
$$

Then sequence $\left\{\phi_{n}\right\}$ is a minimizer sequence for energy $\mathcal{E}_{v}$, even it has not the required charge $N$. We can define a rescaled sequence

$$
\tilde{\phi}_{n}:=a_{n} \phi_{n} \quad \text { with } a_{n}=\sqrt{N / \mathcal{N}\left(\phi_{n}\right)}
$$

we obtain that $\mathcal{N}\left(\tilde{\phi}_{n}\right)=N$. Now, we want to prove that $\tilde{\phi}_{n}$ is also a minimizer sequence of (2).

From Lemma 25, we know $\left\{\phi_{n}\right\}$ is bounded in $H^{1 / 2}(\mathbb{R})$, then we have the following estimation:

$$
\left\|\phi_{n}-\tilde{\phi}_{n}\right\|_{H^{1 / 2}} \leq C\left|1-a_{n}\right| \rightarrow 0, \quad \text { as } n \rightarrow \infty .
$$


By continuity of $\mathcal{E}_{v}: H^{1 / 2}(\mathbb{R}) \rightarrow \mathbb{R}$, we have that

$$
\lim _{n \rightarrow \infty} \mathcal{E}_{v}\left(\tilde{\phi}_{n}\right)=E_{v}(N)
$$

then $\left\{\tilde{\phi}_{n}\right\}$ is a minimizer sequence of (2), under the constraint $\mathcal{N}(\psi)=N$. By Theorem 32 , there exists a subsequence $\left\{\tilde{\phi}_{n_{k}}\right\}$ strongly convergence in $H^{1 / 2}(\mathbb{R})$, up to translations, to a minimizer $\varphi \in S_{v, N}$. From (67), we can replace $\tilde{\phi}_{n}$ by $\phi_{n}$. But, this fact contradicts the second part of (66). Then, weak orbital stability holds.

${ }^{1}$ J. Bellazzini, T. Ozawa, and N. Visciglia, "Ground states for semi-relativistic Schrödinger-Poisson-Slater energies," e-print arXiv:1103.2649.

2 J. P. Borgna, "Stability of periodic nonlinear Schrödinger equation," Nonlinear Anal. Theory, Methods Appl. 69, 4252-4265 (2008).

${ }^{3}$ H. Brezis, Functional Analysis, Sobolev Spaces and Partial Differential Equations (Springer, 2010).

${ }^{4}$ H. Brezis and T. Gallouet, "Nonlinear Schrödinger evolution equations," Nonlinear Anal. 4, 677-671 (1980).

${ }^{5}$ J. Frölich, B. Lars, G. Jonsson, and E. Lenzmann, "Boson stars as solitary waves," Commun. Math. Phys. 274, 1-30 (2007).

${ }^{6}$ E. Lenzmann, "Well-posedness for semi-relativistic Hartree equations of critical type," Math. Phys., Anal. Geom. 10, 43-64 (2007).

${ }^{7}$ E. Lieb and M. Loss, Analysis, 2nd ed., AMS Graduate Studies in Mathematics Vol. 14 (American Mathematical Society, Providence, RI, 2001).

${ }^{8}$ G. Ponce, "On the global well-posedness of the Benjamin-Ono equation," Differential Integral Equations 4, 527-542 (1991).

${ }^{9}$ M. Reed and B. Simon, Methods of Modern Mathematical Physics (Academic, 1972), Vol. 2.

${ }^{10}$ W. A. Strauss, "Existence of solitary waves in higher dimensions," Commun. Math. Phys. 55, 149-162 (1977).

${ }^{11}$ M. Weinstein, "Lyapunov stability of ground states of nonlinear dispersive evolutions equations," Commun. Pure Appl. Math. 39, 51-68 (1986). 